Kragujevac Journal of Mathematics

Volume 38(2) (2014), Pages 283-301.

\title{
NEW RESULTS FOR A SYSTEM OF TWO FRACTIONAL DIFFERENTIAL EQUATIONS INVOLVING $n$ CAPUTO DERIVATIVES
}

\author{
MOHAMED HOUAS ${ }^{1}$ AND ZOUBIR DAHMANI ${ }^{2}$
}

\begin{abstract}
This paper studies a coupled system of two differential equations of arbitrary orders using Caputo approach with $n$ derivatives, $n \in N^{*}, n \neq 1$. New existence and uniqueness results are established using Banach contraction principle. Other existence results are obtained using Schaefer and Krasnoselskii fixed point theorems. Some illustrative examples are also presented.
\end{abstract}

\section{INTRODUCTION}

In recent years, the subject of fractional differential equations has gained a considerable attention and it has emerged as an interesting and popular field of research. For some recent development on this theory, we refer the reader to $[1,2,3,4,5,6,9,19,20]$ and references therein. On the other hand, the study of coupled systems involving fractional differential equations is also important as such systems occur in various problems of applied nature, for instance, see [7, 11, 13, 21, 27]. Some recent results on coupled systems of fractional differential equations on a finite interval can be found in $[8,12,16,17,18,25,26,27]$. In $[8,10,22,23,24]$, the existence and uniqueness of solutions were investigated for a coupled system nonlinear fractional differential equations by using Banach and/or Schauder fixed point theorems.

This paper deals with the existence of solutions for the following problem

$$
\left\{\begin{array}{l}
D^{\alpha_{0}} x(t)=f_{1}\left(t, y(t), D^{\alpha_{1}} y(t), D^{\alpha_{2}} y(t), \ldots, D^{\alpha_{n-1}} y(t)\right), t \in[0,1] \\
D^{\beta_{0}} y(t)=f_{2}\left(t, x(t), D^{\beta_{1}} x(t), D^{\beta_{2}} x(t), \ldots, D^{\beta_{n-1}} x(t)\right), t \in[0,1] \\
x(0)=x^{*}, x^{\prime}(0)=x^{\prime \prime}(0)=\cdots=x^{(n-2)}(0)=0, D^{p} x(1)=\lambda_{1} D^{p} x(\eta) \\
y(0)=y^{*}, y^{\prime}(0)=y^{\prime \prime}(0)=\cdots=y^{(n-2)}(0)=0, D^{q} y(1)=\lambda_{2} D^{q} y(\xi)
\end{array}\right.
$$

Key words and phrases. Caputo derivative, Fixed point, Coupled system, Existence, Uniqueness. 2010 Mathematics Subject Classification. Primary: 34A08.

Received: January 23, 2014

Accepted: Jun 30, 2014. 
where $D^{\alpha_{i}}, D^{\beta_{i}}, i=0,1,2, \ldots, n-1, D^{p}$ and $D^{q}$ denote the Caputo fractional derivatives, with $n-1<\alpha_{n-1}<\cdots<\alpha_{1}<\alpha_{0}<n$ and $n-1<\beta_{n-1}<\cdots<\beta_{1}<$ $\beta_{0}<n, p<\alpha_{0}, q<\beta_{0}, n \in N^{*}, n \neq 1, J=[0,1], \lambda_{1}, \lambda_{2} \neq 0$ are real constants, $x^{*}, y^{*} \in \mathbb{R}, 0<\eta, \xi<1$ are real numbers and $f_{1}, f_{2}$ are two functions which will be specified later.

The rest of this paper is organized as follows: in section 2, we present some preliminaries and lemmas. Section 3 is devoted to existence of solution of the system (1.1). To illustrate our main results, in section 4 , three examples are treated.

\section{Preliminaries}

Let us begin this section with some basic concepts of fractional calculus that will be used throughout this paper.

Definition 2.1. The Riemann-Liouville fractional integral operator of order $\alpha \geq 0$, for a continuous function $f$ on $[0, \infty$ [ is defined as

$$
\begin{aligned}
J^{\alpha} f(t) & =\frac{1}{\Gamma(\alpha)} \int_{0}^{t}(t-\tau)^{\alpha-1} f(\tau) d \tau, \alpha>0, \\
J^{0} f(t) & =f(t),
\end{aligned}
$$

where $\Gamma(\alpha):=\int_{0}^{\infty} e^{-u} u^{\alpha-1} d u$.

Definition 2.2. The fractional derivative of $f \in C^{n}([0, \infty[)$ in the Caputo's sense is defined as

$$
D^{\alpha} f(t)=\frac{1}{\Gamma(n-\alpha)} \int_{0}^{t}(t-\tau)^{n-\alpha-1} f^{(n)}(\tau) d \tau, n-1<\alpha, n \in N^{*} .
$$

For more details, we refer the reader to $[18,20]$.

Let us now introduce the following Banach space

$$
X:=\left\{x: x \in C([0,1], \mathbb{R}) ; D^{\alpha_{1}} x, D^{\alpha_{2}} x, \ldots, D^{\alpha_{n-1}} x \in C([0,1], \mathbb{R})\right\},
$$

endowed with the norm $\|x\|_{X}=\|x\|+\left\|D^{\alpha_{1}} x\right\|+\left\|D^{\alpha_{2}} x\right\|+\cdots+\left\|D^{\alpha_{n-1}} x\right\|$; $\|x\|=\sup _{t \in J}|x(t)|,\left\|D^{\alpha_{1}} x\right\|=\sup _{t \in J}\left|D^{\alpha_{1}} x(t)\right|,\left\|D^{\alpha_{2}} x\right\|=\sup _{t \in J}\left|D^{\alpha_{2}} x(t)\right|, \ldots,\left\|D^{\alpha_{n-1}} x\right\|=$ $\sup _{t \in J}\left|D^{\alpha_{n-1}} x(t)\right|$. Similarly, we can define the space

$$
Y:=\left\{y: y \in C([0,1], \mathbb{R}) ; D^{\beta_{1}} y, D^{\beta_{2}} y, \ldots, D^{\beta_{n-1}} y \in C([0,1], \mathbb{R}),\right.
$$

with the norm $\|y\|_{Y}=\|y\|+\left\|D^{\beta_{1}} y\right\|+\left\|D^{\beta_{2}} y\right\|+\cdots+\left\|D^{\beta_{n-1}} y\right\|$; $\|y\|=\sup _{t \in J}|y(t)|,\left\|D^{\beta_{1}} y\right\|=\sup _{t \in J}\left|D^{\beta_{1}} y(t)\right|,\left\|D^{\beta_{2}} y\right\|=\sup _{t \in J}\left|D^{\beta_{2}} y(t)\right|, \ldots,\left\|D^{\beta_{n-1}} y\right\|=$ $\sup _{t \in J}\left|D^{\beta_{n-1}} y(t)\right|$.

For $(x, y) \in X \times Y$, let $\|(x, y)\|_{X \times Y}=\|x\|_{X}+\|y\|_{Y}$. It is clear that the product space $\left(X \times Y,\|(x, y)\|_{X \times Y}\right)$ is a Banach space.

We give the following lemmas $[14,15]$. 
Lemma 2.1. Let $r, s>0, f \in L_{1}([a, b])$. Then $I^{r} I^{s} f(t)=I^{r+s} f(t), D^{s} I^{s} f(t)=f(t)$, $t \in[a, b]$.

Lemma 2.2. Let $s>r>0, f \in L_{1}([a, b])$. Then $D^{r} I^{s} f(t)=I^{s-r} f(t), t \in[a, b]$.

Also, we present the following two lemmas [14].

Lemma 2.3. For $\alpha>0$, the general solution of the fractional differential equation $D^{\alpha} x(t)=0$ is given by $x(t)=c_{0}+c_{1} t+c_{2} t^{2}+\cdots+c_{n-1} t^{n-1}$, where $c_{i} \in \mathbb{R}, i=$ $0,1,2, \ldots, n-1, n=[\alpha]+1$.

Lemma 2.4. Let $\alpha>0$. Then $J^{\alpha} D^{\alpha} x(t)=x(t)+c_{0}+c_{1} t+c_{2} t^{2}+\cdots+c_{n-1} t^{n-1}$, for some $c_{i} \in \mathbb{R}, i=0,1,2, \ldots, n-1, n=[\alpha]+1$.

The following auxiliary result is crucial to prove our main results.

Lemma 2.5. Let $g \in C([0,1])$. The solution of the equation

$$
D^{\alpha_{0}} x(t)=g(t), t \in J, n-1<\alpha_{0}<n, n>0,
$$

subject to the conditions $x(0)=x^{*}, x^{\prime}(0)=x^{\prime \prime}(0)=\cdots=x^{(n-2)}(0)=0$ and $D^{p} x(1)=\lambda_{1} D^{p} x(\eta)$, is given by

$$
\begin{aligned}
x(t)= & \frac{1}{\Gamma\left(\alpha_{0}\right)} \int_{0}^{t}(t-s)^{\alpha_{0}-1} g(s) d s+x^{*} \\
& -\frac{\Gamma(n-p) t^{n-1}}{\left(1-\lambda_{1} \eta^{n-p-1}\right) \Gamma(n) \Gamma\left(\alpha_{0}-p\right)} \int_{0}^{1}(1-s)^{\alpha_{0}-p-1} g(s) d s \\
& +\frac{\lambda_{1} \Gamma(n-p) t^{n-1}}{\left(1-\lambda_{1} \eta^{n-p-1}\right) \Gamma(n) \Gamma\left(\alpha_{0}-p\right)} \int_{0}^{\eta}(\eta-s)^{\alpha_{0}-p-1} g(s) d s .
\end{aligned}
$$

Proof. We use Lemma 2.3 and Lemma 2.4 to generate the general solution of (2.1). We have

$$
x(t)=\frac{1}{\Gamma\left(\alpha_{0}\right)} \int_{0}^{t}(t-s)^{\alpha_{0}-1} g(s) d s-c_{0}-c_{1} t-c_{2} t^{2}-\cdots-c_{n-1} t^{n-1} .
$$

By $x(0)=x^{*}$, and $x^{\prime}(0)=\cdots=x^{(n-2)}(0)=0$, we can obtain $c_{0}=-x^{*}$ and $c_{1}=c_{2}=\cdots=c_{n-2}=0$.

Thanks to Lemma 2.2, we get

$$
D^{p} x(t)=\frac{1}{\Gamma\left(\alpha_{0}-p\right)} \int_{0}^{t}(t-s)^{\alpha_{0}-p-1} g(s) d s-c_{n-1} \frac{\Gamma(n)}{\Gamma(n-p)} t^{n-p-1} .
$$

Using the condition $D^{p} x(1)=\lambda_{1} D^{p} x(\eta)$, we get

$$
\begin{aligned}
c_{n-1}= & \frac{\Gamma(n-p)}{\left(1-\lambda_{1} \eta^{n-p-1}\right) \Gamma(n) \Gamma\left(\alpha_{0}-p\right)} \int_{0}^{1}(1-s)^{\alpha_{0}-p-1} g(s) d s \\
& -\frac{\lambda_{1} \Gamma(n-p)}{\left(1-\lambda_{1} \eta^{n-p-1}\right) \Gamma(n) \Gamma\left(\alpha_{0}-p\right)} \int_{0}^{\eta}(\eta-s)^{\alpha_{0}-p-1} g(s) d s .
\end{aligned}
$$

Substituting the values of $c_{0}, c_{1}, c_{2}, \ldots, c_{n-2}, c_{n-1}$ in (2.2), we obtain the desired quantity in Lemma 2.5. 


\section{Main Results}

In this section, we present the main results of the paper. For the sake of convenience, let us take.

$$
\begin{aligned}
N_{0} & :=\frac{1}{\Gamma\left(\alpha_{0}+1\right)}+\frac{\Gamma(n-p)\left(1+\left|\lambda_{1}\right| \eta^{\alpha_{0}-p}\right)}{\left|1-\lambda_{1} \eta^{n-p-1}\right| \Gamma(n) \Gamma\left(\alpha_{0}-p+1\right)}, \\
N_{k} & :=\frac{1}{\Gamma\left(\alpha_{0}-\alpha_{k}+1\right)}+\frac{\Gamma(n-p)\left(1+\left|\lambda_{1}\right| \eta^{\alpha_{0}-p}\right)}{\left|1-\lambda_{1} \eta^{n-p-1}\right| \Gamma\left(n-\alpha_{k}\right) \Gamma\left(\alpha_{0}-p+1\right)}, k=1, \ldots, n-1, \\
M_{0} & :=\frac{1}{\Gamma\left(\beta_{0}+1\right)}+\frac{\Gamma(n-q)\left(1+\left|\lambda_{2}\right| \xi^{\beta_{0}-q}\right)}{\left|1-\lambda_{2} \xi^{n-q-1}\right| \Gamma(n) \Gamma\left(\beta_{0}-q+1\right)}, \\
M_{h} & :=\frac{1}{\Gamma\left(\beta_{0}-\beta_{h}+1\right)}+\frac{\Gamma(n-q)\left(1+\left|\lambda_{2}\right| \xi^{\beta_{0}-q}\right)}{\left|1-\lambda_{2} \xi^{n-q-1}\right| \Gamma\left(n-\beta_{h}\right) \Gamma\left(\beta_{0}-q+1\right)}, h=1, \ldots, n-1, \\
\omega & :=\omega_{0}+\omega_{1}+\cdots+\omega_{n-1}, \\
\varpi & :=\varpi_{0}+\varpi_{1}+\cdots+\varpi_{n-1}, \\
\theta & :=\frac{\Gamma(n-p)\left(1+\left|\lambda_{1}\right| \eta^{\alpha_{0}-p}\right)}{\left|1-\lambda_{1} \eta^{n-p-1}\right| \Gamma(n) \Gamma\left(\alpha_{0}-p+1\right)}+\sum_{k=1}^{n-1} \frac{\Gamma(n-p)\left(1+\left|\lambda_{1}\right| \eta^{\alpha_{0}-p}\right)}{\left|1-\lambda_{1} \eta^{n-p-1}\right| \Gamma\left(n-\alpha_{k}\right) \Gamma\left(\alpha_{0}-p+1\right)}, \\
\theta^{\prime} & :=\frac{\Gamma(n-q)\left(1+\left|\lambda_{2}\right| \eta^{\beta_{0}-q}\right)}{\left|1-\lambda_{2} \xi^{n-q-1}\right| \Gamma(n) \Gamma\left(\beta_{0}-q+1\right)}+\sum_{h=1}^{n-1} \frac{\Gamma(n-q)\left(1+\left|\lambda_{2}\right| \xi^{\beta_{0}-q}\right)}{\left|1-\lambda_{2} \xi^{n-q-1}\right| \Gamma\left(n-\beta_{h}\right) \Gamma\left(\beta_{0}-q+1\right)} .
\end{aligned}
$$

$(H 1):$ We also suppose that the functions $f_{1}, f_{2}:[0,1] \times \mathbb{R}^{n} \rightarrow \mathbb{R}$ are continuous. $(H 2)$ : There exist non negative continuous functions $a_{i}, b_{i} \in C([0,1]), i=0, \ldots, n-1$, such that for all $t \in[0,1]$ and $\left(x_{0}, x_{1}, x_{2}, \ldots, x_{n-1}\right),\left(y_{0}, y_{1}, y_{2}, \ldots, y_{n-1}\right) \in \mathbb{R}^{n}$, we have

$$
\begin{aligned}
& \left|f_{1}\left(t, x_{0}, x_{1}, x_{2}, \ldots, x_{n-1}\right)-f_{1}\left(t, y_{0}, y_{1}, y_{2}, \ldots, y_{n-1}\right)\right| \\
& \leq a_{0}(t)\left|x_{0}-y_{0}\right|+a_{1}(t)\left|x_{1}-y_{1}\right|+a_{2}(t)\left|x_{2}-y_{2}\right|+\cdots+a_{n-1}(t)\left|x_{n-1}-y_{n-1}\right|,
\end{aligned}
$$

and

$$
\begin{aligned}
& \left|f_{2}\left(t, x_{0}, x_{1}, x_{2}, \ldots, x_{n-1}\right)-f_{2}\left(t, y_{0}, y_{1}, y_{2}, \ldots, y_{n-1}\right)\right| \\
& \leq b_{0}(t)\left|x_{0}-y_{0}\right|+b_{1}(t)\left|x_{1}-y_{1}\right|+b_{2}(t)\left|x_{2}-y_{2}\right|+\cdots+b_{n-1}(t)\left|x_{n-1}-y_{n-1}\right|,
\end{aligned}
$$

where,

$$
\begin{aligned}
& \omega_{0}=\sup _{t \in J} a_{0}(t), \omega_{1}=\sup _{t \in J} a_{1}(t), \omega_{2}=\sup _{t \in J} a_{2}(t), \ldots, \omega_{n-1}=\sup _{t \in J} a_{n-1}(t), \\
& \varpi_{0}=\sup _{t \in J} b_{0}(t), \varpi_{1}=\sup _{t \in J} b_{1}(t), \varpi_{2}=\sup _{t \in J} b_{2}(t), \ldots, \varpi_{n-1}=\sup _{t \in J} b_{n-1}(t) .
\end{aligned}
$$

$(H 3)$ : There exists non negative functions $l_{1}(t)$ and $l_{2}(t)$ such that

$$
\begin{aligned}
\left|f_{1}\left(t, x_{0}, x_{1}, x_{2}, \ldots, x_{n-1}\right)\right| & \leq l_{1}(t), \\
\left|f_{2}\left(t, x_{0}, x_{1}, x_{2}, \ldots, x_{n-1}\right)\right| & \leq l_{2}(t)
\end{aligned}
$$

for each $t \in J$ and all $x, y \in \mathbb{R}$, with $L_{1}=\sup _{t \in J} l_{1}(t), L_{2}=\sup _{t \in J} l_{2}(t)$.

Our first result is based on Banach contraction principle. It is the following. 
Theorem 3.1. Suppose $\eta^{n-p-1} \neq \frac{1}{\lambda_{1}}, \xi^{n-q-1} \neq \frac{1}{\lambda_{2}}$ and assume that the hypothesis (H2) holds. If

$$
\left(N_{0}+\sum_{k=1}^{n-1} N_{k}\right) \omega+\left(M_{0}+\sum_{h=1}^{n-1} M_{h}\right) \varpi<1,
$$

then the fractional system (1.1) has a unique solution on J.

Proof. Let us define the operator $\phi: X \times Y \rightarrow X \times Y$ by

$$
\phi(x, y)(t):=\left(\phi_{1}(y)(t), \phi_{2}(x)(t)\right)
$$

where, for each $t \in[0,1]$,

$$
\begin{aligned}
& \phi_{1} y(t):=\frac{1}{\Gamma\left(\alpha_{0}\right)} \int_{0}^{t}(t-s)^{\alpha_{0}-1} f_{1}\left(s, y(s), D^{\alpha_{1}} y(s), \ldots, D^{\alpha_{n-1}} y(s)\right) d s+x^{*} \\
& \quad-\frac{\Gamma(n-p) t^{n-1}}{\left(1-\lambda_{1} \eta^{n-p-1}\right) \Gamma(n) \Gamma\left(\alpha_{0}-p\right)} \int_{0}^{1}(1-s)^{\alpha_{0}-p-1} f_{1}\left(s, y(s), D^{\alpha_{1}} y(s), \ldots, D^{\alpha_{n-1}} y(s)\right) d s \\
& \quad+\frac{\lambda_{1} \Gamma(n-p) t^{n-1}}{\left(1-\lambda_{1} \eta^{n-p-1}\right) \Gamma(n) \Gamma\left(\alpha_{0}-p\right)} \int_{0}^{\eta}(\eta-s)^{\alpha_{0}-p-1} f_{1}\left(s, y(s), D^{\alpha_{1}} y(s), \ldots, D^{\alpha_{n-1}} y(s)\right) d s
\end{aligned}
$$

and

$$
\begin{aligned}
& \phi_{2} x(t):=\frac{1}{\Gamma\left(\beta_{0}\right)} \int_{0}^{t}(t-s)^{\beta_{0}-1} f_{2}\left(s, x(s), D^{\beta_{1}} x(s), \ldots, D^{\beta_{n-1}} x(s)\right) d s+y^{*} \\
& \quad-\frac{\Gamma(n-q) t^{n-1}}{\left(1-\lambda_{2} \eta^{n-q-1}\right) \Gamma(n) \Gamma\left(\beta_{0}-q\right)} \int_{0}^{1}(1-s)^{\beta_{0}-q-1} f_{2}\left(s, x(s), D^{\beta_{1}} x(s), \ldots, D^{\beta_{n-1}} x(s)\right) d s \\
& \quad+\frac{\lambda_{2} \Gamma(n-q) t^{n-1}}{\left(1-\lambda_{2} \eta^{n-q-1}\right) \Gamma(n) \Gamma\left(\beta_{0}-q\right)} \int_{0}^{\eta}(\eta-s)^{\beta_{0}-q-1} f_{2}\left(s, x(s), D^{\beta_{1}} x(s), \ldots, D^{\beta_{n-1}} x(s)\right) d s
\end{aligned}
$$

We shall prove that $\phi$ is a contraction mapping.

If we denote $F(s)=\left|\begin{array}{c}f_{1}\left(s, y(s), D^{\alpha_{1}} y(s), \ldots, D^{\alpha_{n-1}} y(s)\right) \\ -f_{1}\left(s, y_{1}(s), D^{\alpha_{1}} y_{1}(s), \ldots, D^{\alpha_{n-1}} y_{1}(s)\right)\end{array}\right|$, then for each $t \in J$ and for $(x, y),\left(x_{1}, y_{1}\right) \in X \times Y$ we have

$$
\begin{aligned}
\left|\phi_{1} y(t)-\phi_{1} y_{1}(t)\right| \leq & \frac{1}{\Gamma\left(\alpha_{0}\right)} \int_{0}^{t}(t-s)^{\alpha_{0}-1} F(s) d s \\
& +\frac{\Gamma(n-p)}{\left|1-\lambda_{1} \eta^{n-p-1}\right| \Gamma(n) \Gamma\left(\alpha_{0}-p\right)} \int_{0}^{1}(1-s)^{\alpha_{0}-p-1} F(s) d s \\
& +\frac{\left|\lambda_{1}\right| \Gamma(n-p)}{\left|1-\lambda_{1} \eta^{n-p-1}\right| \Gamma(n) \Gamma\left(\alpha_{0}-p\right)} \int_{0}^{\eta}(\eta-s)^{\alpha_{0}-p-1} F(s) d s .
\end{aligned}
$$


Thus,

$$
\begin{aligned}
& \left|\phi_{1} y(t)-\phi_{1} y_{1}(t)\right| \leq \frac{\left(\omega_{0}+\omega_{1}+\cdots+\omega_{n-1}\right)\left[\left\|y-y_{1}\right\|+\left\|D^{\alpha_{1}} y-D^{\alpha_{1}} y_{1}\right\|+\cdots+\left\|D^{\alpha_{n-1}} y-D^{\alpha_{n-1}} y_{1}\right\|\right]}{\Gamma\left(\alpha_{0}+1\right)} \\
& \quad+\frac{\Gamma(n-p)\left(\omega_{0}+\omega_{1}+\cdots+\omega_{n-1}\right)\left[\left\|y-y_{1}\right\|+\left\|D^{\alpha_{1}} y-D^{\alpha_{1}} y_{1}\right\|+\cdots+\left\|D^{\alpha_{n-1}} y-D^{\alpha_{n-1}} y_{1}\right\|\right]}{\left|1-\lambda_{1} \eta^{n-p-1}\right| \Gamma(n) \Gamma\left(\alpha_{0}-p+1\right)} \\
& \quad+\frac{\left|\lambda_{1}\right| \Gamma(n-p) \eta^{\alpha_{0}-p}\left(\omega_{0}+\omega_{1}+\cdots+\omega_{n-1}\right)\left[\left\|y-y_{1}\right\|+\left\|D^{\alpha_{1}} y-D^{\alpha_{1}} y_{1}\right\|+\cdots+\left\|D^{\alpha_{n-1}} y-D^{\alpha_{n-1}} y_{1}\right\|\right]}{\left|1-\lambda_{1} \eta^{n-p-1}\right| \Gamma(n) \Gamma\left(\alpha_{0}-p+1\right)}
\end{aligned}
$$

Consequently, we have

$\left|\phi_{1} y(t)-\phi_{1} y_{1}(t)\right| \leq N_{0} \omega\left(\left\|y-y_{1}\right\|+\left\|D^{\alpha_{1}} y-D^{\alpha_{1}} y_{1}\right\|+\cdots+\left\|D^{\alpha_{n-1}} y-D^{\alpha_{n-1}} y_{1}\right\|\right)$, which implies that

$$
\begin{aligned}
\left\|\phi_{1}(y)-\phi_{1}\left(y_{1}\right)\right\| \leq & N_{0} \omega\left(\left\|y-y_{1}\right\|+\left\|D^{\alpha_{1}} y-D^{\alpha_{1}} y_{1}\right\|\right. \\
& \left.+\cdots+\left\|D^{\alpha_{n-1}} y-D^{\alpha_{n-1}} y_{1}\right\|\right) .
\end{aligned}
$$

Similarly,

$$
\begin{aligned}
\left\|\phi_{2}(x)-\phi_{2}\left(x_{1}\right)\right\| \leq & M_{0} \varpi\left(\left\|x-x_{1}\right\|+\left\|D^{\beta_{1}} x-D^{\beta_{1}} x_{1}\right\|\right. \\
& \left.+\cdots+\left\|D^{\beta_{n-1}} x-D^{\beta_{n-1}} x_{1}\right\|\right) .
\end{aligned}
$$

On the other hand, for $F(s)=\left|\begin{array}{c}f_{1}\left(s, y(s), D^{\alpha_{1}} y(s), \ldots, D^{\alpha_{n-1}} y(s)\right) \\ -f_{1}\left(s, y_{1}(s), D^{\alpha_{1}} y_{1}(s), \ldots, D^{\alpha_{n-1}} y_{1}(s)\right)\end{array}\right|$, for all $k=1, \ldots, n-1$ and for each $t \in[0,1]$ we have

$$
\begin{aligned}
\left|D^{\alpha_{k}} \phi_{1} y(t)-D^{\alpha_{k}} \phi_{1} y_{1}(t)\right| \leq & \frac{1}{\Gamma\left(\alpha_{0}-\alpha_{k}\right)} \int_{0}^{t}(t-s)^{\alpha_{0}-\alpha_{k}-1} F(s) d s \\
& +\frac{\Gamma(n-p) t^{n-\alpha_{k}-1}}{\left|1-\lambda_{1} \eta^{n-p-1}\right| \Gamma\left(n-\alpha_{k}\right) \Gamma\left(\alpha_{0}-p\right)} \int_{0}^{1}(1-s)^{\alpha_{0}-p-1} F(s) d s \\
& +\frac{\left|\lambda_{1}\right| \Gamma(n-p) t^{n-\alpha_{k}-1}}{\left|1-\lambda_{1} \eta^{n-p-1}\right| \Gamma\left(n-\alpha_{k}\right) \Gamma\left(\alpha_{0}-p\right)} \int_{0}^{\eta}(\eta-s)^{\alpha_{0}-p-1} F(s) d s .
\end{aligned}
$$

By $(H 2)$, we obtain

$$
\begin{aligned}
& \left|D^{\alpha_{k}} \phi_{1} y(t)-D^{\alpha_{k}} \phi_{1} y_{1}(t)\right| \leq \frac{\left(\omega_{0}+\omega_{1}+\cdots+\omega_{n-1}\right)\left[\left\|y-y_{1}\right\|+\left\|D^{\alpha_{1}} y-D^{\alpha_{1}} y_{1}\right\|+\cdots+\left\|D^{\alpha_{n-1}} y-D^{\alpha_{n-1}} y_{1}\right\|\right]}{\Gamma\left(\alpha_{0}-\alpha_{k}+1\right)} \\
& \quad+\frac{\Gamma(n-p)\left(\omega_{0}+\omega_{1}+\cdots+\omega_{n-1}\right)\left[\left\|y-y_{1}\right\|+\left\|D^{\alpha_{1}} y-D^{\alpha_{1}} y_{1}\right\|+\cdots+\left\|D^{\alpha_{n-1}} y-D^{\alpha_{n-1}} y_{1}\right\|\right]}{\left|1-\lambda_{1} \eta^{n-p-1}\right| \Gamma\left(n-\alpha_{k}\right) \Gamma\left(\alpha_{0}-p+1\right)} \\
& \quad+\frac{\left|\lambda_{1}\right| \Gamma(n-p) \eta^{\alpha_{0}-p}\left(\omega_{0}+\omega_{1}+\cdots+\omega_{n-1}\right)\left[\left\|y-y_{1}\right\|+\left\|D^{\alpha_{1}} y-D^{\alpha_{1}} y_{1}\right\|+\cdots+\left\|D^{\alpha_{n-1}} y-D^{\alpha_{n-1}} y_{1}\right\|\right]}{\left|1-\lambda_{1} \eta^{n-p-1}\right| \Gamma\left(n-\alpha_{k}\right) \Gamma\left(\alpha_{0}-p+1\right)}
\end{aligned}
$$

Hence we have

$$
\begin{aligned}
\left|D^{\alpha_{k}} \phi_{1} y(t)-D^{\alpha_{k}} \phi_{1} y_{1}(t)\right| \leq & N_{k} \omega\left(\left\|y-y_{1}\right\|+\left\|D^{\alpha_{1}} y-D^{\alpha_{1}} y_{1}\right\|\right. \\
& \left.+\cdots+\left\|D^{\alpha_{n-1}} y-D^{\alpha_{n-1}} y_{1}\right\|\right) .
\end{aligned}
$$

Then

$$
\begin{aligned}
\left\|D^{\alpha_{k}} \phi_{1}(y)-D^{\alpha_{k}} \phi_{1}\left(y_{1}\right)\right\| \leq & N_{k} \omega\left(\left\|y-y_{1}\right\|+\left\|D^{\alpha_{1}} y-D^{\alpha_{1}} y_{1}\right\|\right. \\
& \left.+\cdots+\left\|D^{\alpha_{n-1}} y-D^{\alpha_{n-1}} y_{1}\right\|\right) .
\end{aligned}
$$


With the same arguments as before, for each $h=1, \ldots, n-1$, we get

$$
\begin{aligned}
\left\|D^{\beta_{h}} \phi_{2}(x)-D^{\beta_{h}} \phi_{2}\left(x_{1}\right)\right\| \leq & M_{h} \varpi\left(\left\|x-x_{1}\right\|+\left\|D^{\beta_{1}} x-D^{\beta_{1}} x_{1}\right\|\right. \\
& \left.+\cdots+\left\|D^{\beta_{n-1}} x-D^{\beta_{n-1}} x_{1}\right\|\right) .
\end{aligned}
$$

Thanks to (3.2) and (3.4), we obtain

$$
\begin{aligned}
\left\|\phi_{1}(y)-\phi_{1}\left(y_{1}\right)\right\|_{X} & \leq\left(N_{0}+\sum_{k=1}^{n-1} N_{k}\right) \omega\left(\left\|y-y_{1}\right\|+\left\|D^{\alpha_{1}} y-D^{\alpha_{1}} y_{1}\right\|\right. \\
& \left.+\cdots+\left\|D^{\alpha_{n-1}} y-D^{\alpha_{n-1}} y_{1}\right\|\right) .
\end{aligned}
$$

Using (3.3) and (3.5) we can write

$$
\begin{gathered}
\left\|\phi_{2}(x)-\phi_{2}\left(x_{1}\right)\right\|_{Y} \leq( \\
\left.M_{0}+\sum_{h=1}^{n-1} M_{h}\right) \varpi\left(\left\|x-x_{1}\right\|+\left\|D^{\beta_{1}} x-D^{\beta_{1}} x_{1}\right\|\right. \\
\left.+\cdots+\left\|D^{\beta_{n-1}} x-D^{\beta_{n-1}} x_{1}\right\|\right)
\end{gathered}
$$

Combining (3.6) and (3.7), we deduce that

$$
\begin{aligned}
\left\|\phi(x, y)-\phi\left(x_{1}, y_{1}\right)\right\|_{X \times Y} \leq & {\left[\left(N_{0}+\sum_{k=1}^{n-1} N_{k}\right) \omega+\left(M_{0}+\sum_{h=1}^{n-1} M_{h}\right) \varpi\right] } \\
& \times\left\|\left(x-x_{1}, y-y_{1}\right)\right\|_{X \times Y} .
\end{aligned}
$$

Consequently by (3.1) we conclude that $\phi$ is a contraction mapping. As a consequence of Banach contraction principle, we deduce that $\phi$ has a unique fixed point which is the solution of (1.1).

The second main result is given in the following theorem.

Theorem 3.2. Suppose that for all $\eta^{n-p-1} \neq \frac{1}{\lambda_{1}}, \xi^{n-q-1} \neq \frac{1}{\lambda_{2}}$ and assume that the hypotheses (H1)and (H3) are satisfied. Then, the system (1.1) has at least a solution on $J$.

Proof. We use Scheafer's fixed point theorem to prove that $\phi$ has at least one fixed point on $X \times Y$.

Step 1: $\phi$ is continuous on $X \times Y$ : By $(H 1)$ we conclude that the operator $\phi$ is continuous.

Step 2: The operator $\phi$ maps bounded sets into bounded sets in $X \times Y$ : For $\sigma>0$, we take $(x, y) \in B_{\sigma}=\left\{(x, y) \in X \times Y ;\|(x, y)\|_{X \times Y} \leq \sigma\right\}$. For each $t \in J$ and 
$\Delta_{1}=\frac{\Gamma(n-p) t^{n-1}}{\left|1-\lambda_{1} \eta^{n-p-1}\right| \Gamma(n) \Gamma\left(\alpha_{0}-p\right)}$ we have:

$$
\begin{aligned}
\left|\phi_{1} y(t)\right| \leq & \frac{1}{\Gamma\left(\alpha_{0}\right)} \int_{0}^{t}(t-s)^{\alpha_{0}-1}\left|f_{1}\left(s, y(s), D^{\alpha_{1}} y(s), \ldots, D^{\alpha_{n-1}} y(s)\right)\right| d s+\left|x^{*}\right| \\
& +\Delta_{1} \int_{0}^{1}(1-s)^{\alpha_{0}-p-1}\left|f_{1}\left(s, y(s), D^{\alpha_{1}} y(s), \ldots, D^{\alpha_{n-1}} y(s)\right)\right| d s \\
& +\left|\lambda_{1}\right| \Delta_{1} \int_{0}^{\eta}(\eta-s)^{\alpha_{0}-p-1}\left|f_{1}\left(s, y(s), D^{\alpha_{1}} y(s), \ldots, D^{\alpha_{n-1}} y(s)\right)\right| d s .
\end{aligned}
$$

Using (H3), we obtain

$$
\left|\phi_{1} y(t)\right| \leq \sup _{t \in J} l_{1}(t)\left[\frac{1}{\Gamma\left(\alpha_{0}+1\right)}+\frac{\Gamma(n-p)\left(1+\left|\lambda_{1}\right| \eta^{\alpha_{0}-p}\right)}{\left|1-\lambda_{1} \eta^{n-p-1}\right| \Gamma(n) \Gamma\left(\alpha_{0}-p+1\right)}\right]+\left|x^{*}\right| .
$$

Thus, $\left|\phi_{1} y(t)\right| \leq N_{0} \sup _{t \in J} l_{1}(t)+\left|x^{*}\right|, t \in J$, and then

$$
\left\|\phi_{1}(y)\right\| \leq L_{1} N_{0}+\left|x^{*}\right|
$$

Similarly, we can write

$$
\left\|\phi_{2}(x)\right\| \leq L_{2} M_{0}+\left|y^{*}\right|
$$

On the other hand, for all $k=1,2, \ldots, n-1$ and $\Delta_{2}=\frac{\Gamma(n-p) t^{n-\alpha_{k}-1}}{\left|1-\lambda_{1} \eta^{n-p-1}\right| \Gamma\left(n-\alpha_{k}\right) \Gamma\left(\alpha_{0}-p\right)}$ we have

$$
\begin{aligned}
\left|D^{\alpha_{k}} \phi_{1} y(t)\right| \leq & \frac{1}{\Gamma\left(\alpha_{0}-\alpha_{k}\right)} \int_{0}^{t}(t-s)^{\alpha_{0}-\alpha_{k}-1}\left|f_{1}\left(s, y(s), D^{\alpha_{1}} y(s), \ldots, D^{\alpha_{n-1}} y(s)\right)\right| d s \\
& +\Delta_{2} \int_{0}^{1}(1-s)^{\alpha_{0}-p-1}\left|f_{1}\left(s, y(s), D^{\alpha_{1}} y(s), \ldots, D^{\alpha_{n-1}} y(s)\right)\right| d s \\
& +\left|\lambda_{1}\right| \Delta_{2} \int_{0}^{\eta}(\eta-s)^{\alpha_{0}-p-1}\left|f_{1}\left(s, y(s), D^{\alpha_{1}} y(s), \ldots, D^{\alpha_{n-1}} y(s)\right)\right| d s .
\end{aligned}
$$

By (H3), we obtain

$$
\left\|D^{\alpha_{k}} \phi_{1}(y)\right\| \leq L_{1}\left[\frac{1}{\Gamma\left(\alpha_{0}-\alpha_{k}+1\right)}+\frac{\Gamma(n-p)\left(1+\left|\lambda_{1}\right| \eta^{\alpha_{0}-p}\right)}{\left|1-\lambda_{1} \eta^{n-p-1}\right| \Gamma\left(n-\alpha_{k}\right) \Gamma\left(\alpha_{0}-p+1\right)}\right],
$$

and for all $h=1,2, \ldots, n-1$,

$$
\left\|D^{\beta_{h}} \phi_{2}(x)\right\| \leq L_{2}\left[\frac{1}{\Gamma\left(\beta_{0}-\beta_{h}+1\right)}+\frac{\Gamma(n-q)\left(1+\left|\lambda_{2}\right| \xi^{\beta_{0}-q}\right)}{\left|1-\lambda_{2} \xi^{n-q-1}\right| \Gamma\left(n-\beta_{h}\right) \Gamma\left(\beta_{0}-q+1\right)}\right] .
$$

Combining (3.8) and (3.10), yields to

$$
\left\|\phi_{1}(y)\right\|_{X} \leq L_{1}\left(N_{0}+\sum_{k=1}^{n-1} N_{k}\right)+\left|x^{*}\right| .
$$


Similarly, it follows from (3.9) and (3.11) that

$$
\left\|\phi_{2}(x)\right\|_{Y} \leq L_{2}\left(M_{0}+\sum_{h=1}^{n-1} M_{h}\right)+\left|y^{*}\right| .
$$

Thanks to (3.12) and (3.13), we have

$$
\|\phi(x, y)\|_{X \times Y} \leq L_{1}\left(N_{0}+\sum_{k=1}^{n-1} N_{k}\right)+L_{2}\left(M_{0}+\sum_{h=1}^{n-1} M_{h}\right)+\left|x^{*}\right|+\left|y^{*}\right| .
$$

Consequently $\|\phi(x, y)\|_{X \times Y}<\infty$.

Step 3: Now we show that $\phi$ is equi-continuous on $J$.

Let us take $(x, y) \in B_{\sigma}, t_{1}, t_{2} \in J$, such that $t_{1}<t_{2}$. Thanks to $(H 3)$, we can write:

$$
\begin{aligned}
\left|\phi_{1} y\left(t_{2}\right)-\phi_{1} y\left(t_{1}\right)\right| \leq & \frac{\sup _{t \in J} l_{1}(t)}{\Gamma\left(\alpha_{0}\right)} \int_{0}^{t_{1}}\left[\left(t_{2}-s\right)^{\alpha_{0}-1}-\left(t_{1}-s\right)^{\alpha_{0}-1}\right] d s \\
& +\frac{\sup _{t \in J} l_{1}(t)}{\Gamma\left(\alpha_{0}\right)} \int_{t_{1}}^{t_{2}}\left(t_{2}-s\right)^{\alpha_{0}-1} d s \\
& +\frac{\sup _{t \in J} l_{1}(t) \Gamma(n-p)\left(t_{1}^{n-1}-t_{2}^{n-1}\right)}{\left|1-\lambda_{1} \eta^{n-p-1}\right| \Gamma(n) \Gamma\left(\alpha_{0}-p\right)} \int_{0}^{1}(1-s)^{\alpha_{0}-p-1} d s \\
& +\frac{\sup _{t \in J} l_{1}(t)\left|\lambda_{1}\right| \Gamma(n-p)\left(t_{2}^{n-1}-t_{1}^{n-1}\right)}{\left|1-\lambda_{1} \eta^{n-p-1}\right| \Gamma(n) \Gamma\left(\alpha_{0}-p\right)} \int_{0}^{\eta}(\eta-s)^{\alpha_{0}-p-1} d s .
\end{aligned}
$$

Thus,

$$
\begin{aligned}
\left|\phi_{1} y\left(t_{2}\right)-\phi_{1} y\left(t_{1}\right)\right| \leq & \frac{L_{1}}{\Gamma\left(\alpha_{0}+1\right)}\left(t_{1}^{\alpha_{0}}-t_{2}^{\alpha_{0}}\right)+\frac{2 L_{1}}{\Gamma\left(\alpha_{0}+1\right)}\left(t_{2}-t_{1}\right)^{\alpha_{0}} \\
& +\frac{L_{1} \Gamma(n-p)}{\left|1-\lambda_{1} \eta^{n-p-1}\right| \Gamma(n) \Gamma\left(\alpha_{0}-p+1\right)}\left(t_{1}^{n-1}-t_{2}^{n-1}\right) \\
& +\frac{L_{1}\left|\lambda_{1}\right| \Gamma(n-p) \eta_{0}-p}{\left|1-\lambda_{1} \eta^{n-p-1}\right| \Gamma(n) \Gamma\left(\alpha_{0}-p+1\right)}\left(t_{2}^{n-1}-t_{1}^{n-1}\right) .
\end{aligned}
$$

Analogously, we can write

$$
\begin{aligned}
\left|\phi_{2} x\left(t_{2}\right)-\phi_{2} x\left(t_{1}\right)\right| \leq & \frac{L_{2}}{\Gamma\left(\beta_{0}+1\right)}\left(t_{1}^{\beta_{0}}-t_{2}^{\beta_{0}}\right)+\frac{2 L_{2}}{\Gamma\left(\beta_{0}+1\right)}\left(t_{2}-t_{1}\right)^{\beta_{0}} \\
& +\frac{L_{2} \Gamma(n-q)}{\left|1-\lambda_{2} \xi^{n-q-1}\right| \Gamma(n) \Gamma\left(\beta_{0}-q+1\right)}\left(t_{1}^{n-1}-t_{2}^{n-1}\right) \\
& +\frac{L_{2}\left|\lambda_{2}\right| \Gamma(n-q) \eta^{\beta_{0}-q}}{\left|1-\lambda_{2} \xi^{n-q-1}\right| \Gamma(n) \Gamma\left(\beta_{0}-q+1\right)}\left(t_{2}^{n-1}-t_{1}^{n-1}\right) .
\end{aligned}
$$

On the other hand, for all $k=1,2 \ldots, n-1$,

$\left|D^{\alpha_{k}} \phi_{1} y\left(t_{2}\right)-D^{\alpha_{k}} \phi_{1} y\left(t_{1}\right)\right| \leq \frac{L_{1}}{\Gamma\left(\alpha_{0}-\alpha_{k}+1\right)}\left(t_{1}^{\alpha_{0}-\alpha_{k}}-t_{2}^{\alpha_{0}-\alpha_{k}}\right)+\frac{2 L_{1}}{\Gamma\left(\alpha_{0}-\alpha_{k}+1\right)}\left(t_{2}-t_{1}\right)^{\alpha_{0}-\alpha_{k}}$

$$
\begin{aligned}
& +\frac{L_{1} \Gamma(n-p)}{\left|1-\lambda_{1} \eta^{n-p-1}\right| \Gamma\left(n-\alpha_{k}\right) \Gamma\left(\alpha_{0}-p+1\right)}\left(t_{1}^{n-\alpha_{k}-1}-t_{2}^{n-\alpha_{k}-1}\right) \\
& +\frac{L_{1}\left|\lambda_{1}\right| \Gamma(n-p) \eta^{\alpha_{0}-p}}{\left|1-\lambda_{1} \eta^{n-p-1}\right| \Gamma\left(n-\alpha_{k}\right) \Gamma\left(\alpha_{0}-p+1\right)}\left(t_{2}^{n-\alpha_{k}-1}-t_{1}^{n-\alpha_{k}-1}\right),
\end{aligned}
$$


and for all $h=1,2 \ldots, n-1$,

$$
\begin{aligned}
\left|D^{\beta_{h}} \phi_{2} x\left(t_{2}\right)-D^{\beta_{h}} \phi_{2} x\left(t_{1}\right)\right| \leq & \frac{L_{2}}{\Gamma\left(\beta_{0}-\beta_{h}+1\right)}\left(t_{1}^{\beta_{0}-\beta_{h}}-t_{2}^{\beta_{0}-\beta_{h}}\right)+\frac{2 L_{2}}{\Gamma\left(\beta_{0}-\beta_{h}+1\right)}\left(t_{2}-t_{1}\right)^{\beta_{0}-\beta_{h}} \\
& +\frac{L_{2} \Gamma(n-q)}{\left|1-\lambda_{2} \xi^{n-q-1}\right| \Gamma\left(n-\beta_{h}\right) \Gamma\left(\beta_{0}-q+1\right)}\left(t_{1}^{n-\beta_{h}-1}-t_{2}^{n-\beta_{h}-1}\right) \\
& +\frac{L_{2}\left|\lambda_{2}\right| \Gamma(n-q) \eta^{\beta_{0}-q}}{\left|1-\lambda_{2} \xi^{n-q-1}\right| \Gamma\left(n-\beta_{h}\right) \Gamma\left(\beta_{0}-q+1\right)}\left(t_{2}^{n-\beta_{h}-1}-t_{1}^{n-\beta_{h}-1}\right) .
\end{aligned}
$$

By (3.14), (3.15) , (3.16) and (3.17), we can state that $\left\|\phi(x, y)\left(t_{2}\right)-\phi(x, y)\left(t_{1}\right)\right\| \rightarrow$ 0 as $t_{2} \rightarrow t_{1}$. By Arzela-Ascoli theorem, we conclude that $\phi$ is completely continuous operator.

Step 4: Finally, we show that the set $\Omega$ defined by

$$
\Omega=\{(x, y) \in X \times Y,(x, y)=\mu \phi(x, y), 0<\mu<1\},
$$

is bounded.

Let $(x, y) \in \Omega$, then $(x, y)=\mu \phi(x, y)$, for some $0<\mu<1$. Thus, for each $t \in J$, we have $x(t)=\mu \phi_{1} y(t), y(t)=\mu \phi_{2} x(t)$. Then for $\Delta_{1}=\frac{\Gamma(n-p) t^{n-1}}{\left|1-\lambda_{1} \eta^{n-p-1}\right| \Gamma(n) \Gamma\left(\alpha_{0}-p\right)}$

$$
\begin{aligned}
\frac{1}{\mu}|x(t)| \leq & \frac{1}{\Gamma\left(\alpha_{0}\right)} \int_{0}^{t}(t-s)^{\alpha_{0}-1}\left|f_{1}\left(s, y(s), D^{\alpha_{1}} y(s), \ldots, D^{\alpha_{n-1}} y(s)\right)\right| d s+\left|x^{*}\right| \\
& +\Delta_{1} \int_{0}^{1}(1-s)^{\alpha_{0}-p-1}\left|f_{1}\left(s, y(s), D^{\alpha_{1}} y(s), \ldots, D^{\alpha_{n-1}} y(s)\right)\right| d s \\
& +\left|\lambda_{1}\right| \Delta_{1} \int_{0}^{\eta}(\eta-s)^{\alpha_{0}-p-1}\left|f_{1}\left(s, y(s), D^{\alpha_{1}} y(s), \ldots, D^{\alpha_{n-1}} y(s)\right)\right| d s .
\end{aligned}
$$

Thanks to $(H 3)$, we can write

$$
\frac{1}{\mu}|x(t)| \leq \frac{\sup _{t \in J} l_{1}(t)}{\Gamma\left(\alpha_{0}+1\right)}+\left|x^{*}\right|+\frac{\sup _{t \in J} l_{1}(t) \Gamma(n-p)}{\left|1-\lambda_{1} \eta^{n-p-1}\right| \Gamma(n) \Gamma\left(\alpha_{0}-p+1\right)}+\frac{\sup _{t \in J} l_{1}(t)\left|\lambda_{1}\right| \eta^{\alpha_{0}-p} \Gamma(n-p)}{\left|1-\lambda_{1} \eta^{n-p-1}\right| \Gamma(n) \Gamma\left(\alpha_{0}-p+1\right)} .
$$

Therefore,

$$
|x(t)| \leq \mu \sup _{t \in J} l_{1}(t)\left[\frac{1}{\Gamma\left(\alpha_{0}+1\right)}+\frac{\Gamma(n-p)\left(1+\left|\lambda_{1}\right| \eta^{\alpha_{0}-p}\right)}{\left|1-\lambda_{1} \eta^{n-p-1}\right| \Gamma(n) \Gamma\left(\alpha_{0}-p+1\right)}\right]+\left|x^{*}\right| .
$$

Hence, $|x(t)| \leq \mu L_{1} N_{0}+\left|x^{*}\right|, t \in J$, which implies that,

$$
\|x\| \leq \mu L_{1} N_{0}+\left|x^{*}\right| .
$$

Analogously, we have

$$
\|y\| \leq \mu L_{2} M_{0}+\left|y^{*}\right| .
$$

On the other hand, for all $k,=1,2, \ldots, n-1$, we have

$$
\left|D^{\alpha_{k}} x(t)\right| \leq \mu \sup _{t \in J} l_{1}(t)\left[\frac{1}{\Gamma\left(\alpha_{0}-\alpha_{k}+1\right)}+\frac{\Gamma(n-p)\left(1+\left|\lambda_{1}\right| \eta^{\alpha_{0}-p}\right)}{\left|1-\lambda_{1} \eta^{n-p-1}\right| \Gamma\left(n-\alpha_{k}\right) \Gamma\left(\alpha_{0}-p+1\right)}\right], t \in J,
$$

Thus,

$$
\left\|D^{\alpha_{k}} x(t)\right\| \leq \mu L_{1} N_{k}
$$


and for all $h,=1,2, \ldots, n-1$,

$$
\left\|D^{\beta_{h}} y(t)\right\| \leq \mu L_{2} M_{h}
$$

From (3.18) and (3.20), we get

$$
\|x\|_{X} \leq \mu L_{1}\left(N_{0}+\sum_{k=1}^{n-1} N_{k}\right)+\left|x^{*}\right| .
$$

Analogously, by (3.19) and (3.21), yields

$$
\|y\|_{Y} \leq \mu L_{2}\left(M_{0}+\sum_{h=1}^{n-1} M_{h}\right)+\left|y^{*}\right|
$$

It follows from (3.22) and (3.23), that

$$
\|(x, y)\|_{X \times Y} \leq \mu\left[L_{1}\left(N_{0}+\sum_{k=1}^{n-1} N_{k}\right)+L_{2}\left(M_{0}+\sum_{h=1}^{n-1} M_{h}\right)\right]+\left|x^{*}\right|+\left|y^{*}\right| .
$$

Hence, $\|\phi(x, y)\|_{X \times Y}<\infty$.

This shows that $\Omega$ is bounded.

As consequence of Schaefer's fixed point theorem, we deduce that $\phi$ at least a fixed point, which is a solution of the fractional differential system (1.1).

Our third result is based on Krasnoselskii theorem [14].

Theorem 3.3. Let $\eta^{n-p-1} \neq \frac{1}{\lambda_{1}}, \xi^{n-q-1} \neq \frac{1}{\lambda_{2}}$. Suppose that (H1), (H2) and (H3) are satisfied, such that

$$
\left(\frac{1}{\Gamma\left(\alpha_{0}+1\right)}+\sum_{k=1}^{n-1} \frac{1}{\Gamma\left(\alpha_{0}-\alpha_{k}+1\right)}\right) \omega+\left(\frac{1}{\Gamma\left(\beta_{0}+1\right)}+\sum_{h=1}^{n-1} \frac{1}{\Gamma\left(\beta_{0}-\beta_{h}+1\right)}\right) \varpi<1 .
$$

If there exist $\delta \in \mathbb{R}$ such that

$$
L_{1}\left(N_{0}+\sum_{k=1}^{n-1} N_{k}\right)+L_{2}\left(M_{0}+\sum_{h=1}^{n-1} M_{h}\right)+\left|y^{*}\right|+\left|x^{*}\right| \leq \delta,
$$

then the fractional system (1.1) has at least one solution on J.

Proof. We shall prove that $\phi$ has at least a fixed point on $X \times Y$.

Suppose that $L_{1}\left(N_{0}+\sum_{k=1}^{n-1} N_{k}\right)+L_{2}\left(M_{0}+\sum_{h=1}^{n-1} M_{h}\right)+\left|y^{*}\right|+\left|x^{*}\right| \leq \delta$ and let us take

$$
\phi(x, y)(t):=T(x, y)(t)+R(x, y)(t)=\left(T_{1} y(t), T_{2} x(t)\right)+\left(R_{1} y(t), R_{2} x(t)\right),
$$

where

$$
\begin{aligned}
& T_{1} y(t):=\frac{1}{\Gamma\left(\alpha_{0}\right)} \int_{0}^{t}(t-s)^{\alpha_{0}-1} f_{1}\left(s, y(s), D^{\alpha_{1}} y(s), \ldots, D^{\alpha_{n-1}} y(s)\right) d s+\left|x^{*}\right|, \\
& T_{2} x(t):=\frac{1}{\Gamma\left(\beta_{0}\right)} \int_{0}^{t}(t-s)^{\beta_{0}-1} f_{2}\left(s, x(s), D^{\beta_{1}} x(s), \ldots, D^{\beta_{n-1}} x(s)\right) d s+\left|y^{*}\right|,
\end{aligned}
$$


and

$$
\begin{aligned}
& R_{1} y(t):= \\
& \quad-\frac{\Gamma(n-p) t^{n-1}}{\left(1-\lambda_{1} \eta^{n-p-1}\right) \Gamma(n) \Gamma\left(\alpha_{0}-p\right)} \int_{0}^{1}(1-s)^{\alpha_{0}-p-1} f_{1}\left(s, y(s), D^{\alpha_{1}} y(s), \ldots, D^{\alpha_{n-1}} y(s)\right) d s \\
& \quad+\frac{\lambda_{1} \Gamma(n-p) t^{n-1}}{\left(1-\lambda_{1} \eta^{n-p-1}\right) \Gamma(n) \Gamma\left(\alpha_{0}-p\right)} \int_{0}^{\eta}(\eta-s)^{\alpha_{0}-p-1} f_{1}\left(s, y(s), D^{\alpha_{1}} y(s), \ldots, D^{\alpha_{n-1}} y(s)\right) d s, \\
& R_{2} x(t):= \\
& \quad-\frac{\Gamma(n-q) t^{n-1}}{\left(1-\lambda_{2} \xi^{n-q-1}\right) \Gamma(n) \Gamma\left(\beta_{0}-q\right)} \int_{0}^{1}(1-s)^{\beta_{0}-q-1} f_{2}\left(s, x(s), D^{\beta_{1}} x(s), \ldots, D^{\beta_{n-1}} x(s)\right) d s \\
& \quad+\frac{\lambda_{2} \Gamma(n-p) t^{n-1}}{\left(1-\lambda_{2} \xi^{n-q-1}\right) \Gamma(n) \Gamma\left(\beta_{0}-q\right)} \int_{0}^{1} \int_{0}^{\eta}(\eta-s)^{\beta_{0}-q-1} f_{2}\left(s, x(s), D^{\beta_{1}} x(s), \ldots, D^{\beta_{n-1}} x(s)\right) d s .
\end{aligned}
$$

The proof will be given in the following steps:

$\left(1^{*}:\right)$ We shall prove that for any $(x, y),\left(x_{1}, y_{1}\right) \in B_{\delta}$, then $T(x, y)(t)+R\left(x_{1}, y_{1}\right)(t) \in$ $B_{\delta}$, such that $B_{\delta}=\left\{(x, y) \in X \times Y ;\|(x, y)\|_{X \times Y} \leq \delta\right\}$.

For any $(x, y),\left(x_{1}, y_{1}\right) \in B_{\delta}$ and for each $t \in J$, we have

$$
\begin{aligned}
& \left|T_{1} y(t)+R_{1} y_{1}(t)\right| \leq \frac{1}{\Gamma\left(\alpha_{0}\right)} \int_{0}^{t}(t-s)^{\alpha_{0}-1}\left|f_{1}\left(s, y(s), D^{\alpha_{1}} y(s), \ldots, D^{\alpha_{n-1}} y(s)\right)\right| d s+\left|x^{*}\right| \\
& \quad+\frac{\Gamma(n-p) t^{n-1}}{\left|1-\lambda_{1} \eta^{n-p-1}\right| \Gamma(n) \Gamma\left(\alpha_{0}-p\right)} \int_{0}^{1}(1-s)^{\alpha_{0}-p-1}\left|f_{1}\left(s, y_{1}(s), D^{\alpha_{1}} y_{1}(s), \ldots, D^{\alpha_{n-1}} y_{1}(s)\right)\right| d s \\
& \quad+\frac{\lambda_{1} \Gamma(n-p) t^{n-1}}{\left|1-\lambda_{1} \eta^{n-p-1}\right| \Gamma(n) \Gamma\left(\alpha_{0}-p\right)} \int_{0}^{\eta}(\eta-s)^{\alpha_{0}-p-1}\left|f_{1}\left(s, y_{1}(s), D^{\alpha_{1}} y_{1}(s), \ldots, D^{\alpha_{n-1}} y_{1}(s)\right)\right| d s
\end{aligned}
$$

Using (H3), we obtain

$$
\left|T_{1} y(t)+R_{1} y_{1}(t)\right| \leq \sup _{t \in J} l_{1}(t)\left[\frac{1}{\Gamma\left(\alpha_{0}+1\right)}+\frac{\Gamma(n-p)\left(1+\left|\lambda_{1}\right| \eta^{\alpha_{0}-p}\right)}{\left|1-\lambda_{1} \eta^{n-p-1}\right| \Gamma(n) \Gamma\left(\alpha_{0}-p+1\right)}\right]+\left|x^{*}\right| .
$$

Consequently, $\left|T_{1} y(t)+R_{1} y_{1}(t)\right| \leq N_{0} \sup _{t \in J} l_{1}(t)+\left|x^{*}\right|, t \in J$. Thus,

$$
\left\|T_{1}(y)+R_{1}\left(y_{1}\right)\right\| \leq L_{1} N_{0}+\left|x^{*}\right| .
$$

On the other hand, for all $k=1,2, \ldots, n-1$, we have

$$
\left|D^{\alpha_{k}} T_{1} y(t)+D^{\alpha_{k}} R_{1} y_{1}(t)\right| \leq \sup _{t \in J} l_{1}(t)\left[\frac{1}{\Gamma\left(\alpha_{0}-\alpha_{k}+1\right)}+\frac{\Gamma(n-p)\left(1+\left|\lambda_{1}\right| \eta^{\alpha_{0}-p}\right)}{\left|1-\lambda_{1} \eta^{n-p-1}\right| \Gamma\left(n-\alpha_{k}\right) \Gamma\left(\alpha_{0}-p+1\right)}\right],
$$

Hence,

$$
\left\|D^{\alpha_{k}} T_{1} y(t)+D^{\alpha_{k}} R_{1} y_{1}(t)\right\| \leq L_{1} N_{k} .
$$

Combining (3.25) and (3.26), yields

$$
\left\|T_{1}(y)+R_{1}\left(y_{1}\right)\right\|_{X} \leq L_{1}\left(N_{0}+\sum_{k=1}^{n-1} N_{k}\right)+\left|x^{*}\right| .
$$


Analogously, for all $h=1,2, \ldots, n-1$, we have

$$
\left\|T_{2}(x)+R_{2}\left(x_{1}\right)\right\|_{Y} \leq L_{2}\left(M_{0}+\sum_{h=1}^{n-1} M_{h}\right)+\left|y^{*}\right|
$$

Hence, it follows from (3.27) and (3.28) that

$$
\left\|T(x, y)+R\left(x_{1}, y_{1}\right)\right\|_{X \times Y} \leq L_{1}\left(N_{0}+\sum_{k=1}^{n-1} N_{k}\right)+L_{2}\left(M_{0}+\sum_{h=1}^{n-1} M_{h}\right)+\left|y^{*}\right|+\left|x^{*}\right| \leq \delta .
$$

Therefore, $\left\|T(x, y)+R\left(x_{1}, y_{1}\right)\right\|_{X \times Y} \in B_{\delta}$.

$\left(2^{*}:\right)$ We shall prove that $R$ is continuous and compact. Note that $R$ is continuous on $X \times Y$ in view of the continuity of $f_{1}$ and $f_{2}$ (hypothesis $(H 1)$ ).

$\left(a^{*}\right)$ : Now, we prove that $R$ maps bounded sets into bounded sets of $X \times Y$.

For $(x, y) \in B_{\delta}, \Delta_{1}=\frac{\Gamma(n-p) t^{n-1}}{\left|1-\lambda_{1} \eta^{n-p-1}\right| \Gamma(n) \Gamma\left(\alpha_{0}-p\right)}$ and for each $t \in J$, we have

$$
\begin{aligned}
\left|R_{1} y(t)\right| \leq & \Delta_{1} \int_{0}^{1}(1-s)^{\alpha_{0}-p-1}\left|f_{1}\left(s, y(s), D^{\alpha_{1}} y(s), \ldots, D^{\alpha_{n-1}} y(s)\right)\right| d s \\
& +\left|\lambda_{1}\right| \Delta_{1} \int_{0}^{\eta}(\eta-s)^{\alpha_{0}-p-1}\left|f_{1}\left(s, y(s), D^{\alpha_{1}} y(s), \ldots, D^{\alpha_{n-1}} y(s)\right)\right| d s .
\end{aligned}
$$

Thanks to (H3), we obtain

$$
\left|R_{1} y(t)\right| \leq \frac{\sup _{t \in J} l_{1}(t) \Gamma(n-p)\left(1+\left|\lambda_{1}\right| \eta^{\alpha_{0}-p}\right)}{\left|1-\lambda_{1} \eta^{n-p-1}\right| \Gamma(n) \Gamma\left(\alpha_{0}-p+1\right)}, t \in J
$$

Therefore,

$$
\left\|R_{1}(y)\right\| \leq \frac{L_{1} \Gamma(n-p)\left(1+\left|\lambda_{1}\right| \eta^{\alpha_{0}-p}\right)}{\left|1-\lambda_{1} \eta^{n-p-1}\right| \Gamma(n) \Gamma\left(\alpha_{0}-p+1\right)} .
$$

On the other hand, for all $k=1,2, \ldots, n-1$, we have

$$
\left\|D^{\alpha_{k}} R_{1}(y)\right\| \leq \frac{L_{1} \Gamma(n-p)\left(1+\left|\lambda_{1}\right| \eta^{\alpha_{0}-p}\right)}{\left|1-\lambda_{1} \eta^{n-p-1}\right| \Gamma\left(n-\alpha_{k}\right) \Gamma\left(\alpha_{0}-p+1\right)} .
$$

Using (3.29) and (3.30), we have

$$
\left\|R_{1}(y)\right\|_{X} \leq L_{1}\left(\frac{\Gamma(n-p)\left(1+\left|\lambda_{1}\right| \eta^{\alpha_{0}-p}\right)}{\left|1-\lambda_{1} \eta^{n-p-1}\right| \Gamma(n) \Gamma\left(\alpha_{0}-p+1\right)}+\sum_{k=1}^{n-1} \frac{\Gamma(n-p)\left(1+\left|\lambda_{1}\right| \eta^{\alpha_{0}-p}\right)}{\left|1-\lambda_{1} \eta^{n-p-1}\right| \Gamma\left(n-\alpha_{k}\right) \Gamma\left(\alpha_{0}-p+1\right)}\right) .
$$

Similarly, for all $h=1,2, \ldots, n-1$,

$$
\left\|R_{2}(x)\right\|_{Y} \leq L_{2}\left(\frac{\Gamma(n-q)\left(1+\left|\lambda_{2}\right| \eta^{\beta_{0}-q}\right)}{\left|1-\lambda_{2} \xi^{n-q-1}\right| \Gamma(n) \Gamma\left(\beta_{0}-q+1\right)}+\sum_{h=1}^{n-1} \frac{\Gamma(n-q)\left(1+\left|\lambda_{2}\right| \xi^{\beta_{0}-q}\right)}{\left|1-\lambda_{2} \xi^{n-q-1}\right| \Gamma\left(n-\beta_{h}\right) \Gamma\left(\beta_{0}-q+1\right)}\right) .
$$

It follows from (3.31) and (3.32) that $\|R(x, y)\|_{X \times Y} \leq L_{1} \theta+L_{2} \theta^{\prime}<\infty$.

$\left(b^{*}\right)$ : Now, we show that $R$ is equi-continuous on $J$. 
Let $t_{1}, t_{2} \in J$, such that $t_{2}<t_{1}$ and $(x, y) \in B_{\delta}$. Then by (H3), we obtain:

$$
\begin{aligned}
\left|R_{1} y\left(t_{1}\right)-R_{1} y\left(t_{2}\right)\right| \leq & \frac{\sup _{t \in J} l_{1}(t) \Gamma(n-p)\left(t_{2}^{n-1}-t_{1}^{n-1}\right)}{\left|1-\lambda_{1} \eta^{n-p-1}\right| \Gamma(n) \Gamma\left(\alpha_{0}-p\right)} \int_{0}^{1}(1-s)^{\alpha_{0}-p-1} d s \\
& +\frac{\sup _{t \in J} l_{1}(t)\left|\lambda_{1}\right| \Gamma(n-p)\left(t_{1}^{n-1}-t_{2}^{n-1}\right)}{\left|1-\lambda_{1} \eta^{n-p-1}\right| \Gamma(n) \Gamma\left(\alpha_{0}-p\right)} \int_{0}^{\eta}(\eta-s)^{\alpha_{0}-p-1} d s .
\end{aligned}
$$

Thus,

$$
\begin{aligned}
\left|R_{1} y\left(t_{1}\right)-R_{1} y\left(t_{2}\right)\right| \leq & \frac{L_{1} \Gamma(n-p)}{\left|1-\lambda_{1} \eta^{n-p-1}\right| \Gamma(n) \Gamma\left(\alpha_{0}-p+1\right)}\left(t_{2}^{n-1}-t_{1}^{n-1}\right) \\
& +\frac{L_{1}\left|\lambda_{1}\right| \Gamma(n-p) \eta_{0}-p}{\left|1-\lambda_{1} \eta^{n-p-1}\right| \Gamma(n) \Gamma\left(\alpha_{0}-p+1\right)}\left(t_{1}^{n-1}-t_{2}^{n-1}\right) .
\end{aligned}
$$

In the same way, we have

$$
\begin{aligned}
\left|R_{2} x\left(t_{1}\right)-R_{2} x\left(t_{2}\right)\right| \leq & \frac{L_{2} \Gamma(n-q)}{\left|1-\lambda_{2} \xi^{n-q-1}\right| \Gamma(n) \Gamma\left(\beta_{0}-q+1\right)}\left(t_{2}^{n-1}-t_{1}^{n-1}\right) \\
& +\frac{L_{2}\left|\lambda_{2}\right| \Gamma(n-q) \xi_{0}-q}{\left|1-\lambda_{2} \xi^{n-q-1}\right| \Gamma(n) \Gamma\left(\beta_{0}-q+1\right)}\left(t_{1}^{n-1}-t_{2}^{n-1}\right) .
\end{aligned}
$$

On the other hand, for all $k=1,2 \ldots, n-1$,

$$
\begin{aligned}
\left|D^{\alpha_{k}} R_{1} y\left(t_{1}\right)-D^{\alpha_{k}} R_{1} y\left(t_{2}\right)\right| \leq & \frac{L_{1} \Gamma(n-p)}{\left|1-\lambda_{1} \eta^{n-p-1}\right| \Gamma\left(n-\alpha_{k}\right) \Gamma\left(\alpha_{0}-p+1\right)}\left(t_{2}^{n-\alpha_{k}-1}-t_{1}^{n-\alpha_{k}-1}\right) \\
& +\frac{L_{1}\left|\lambda_{1}\right| \Gamma(n-p) \eta^{\alpha_{0}-p}}{\left|1-\lambda_{1} \eta^{n-p-1}\right| \Gamma\left(n-\alpha_{k}\right) \Gamma\left(\alpha_{0}-p+1\right)}\left(t_{1}^{n-\alpha_{k}-1}-t_{2}^{n-\alpha_{k}-1}\right),
\end{aligned}
$$

and for all $h=1,2 \ldots, n-1$,

$$
\begin{aligned}
\left|D^{\beta_{h}} R_{2} x\left(t_{1}\right)-D^{\beta_{h}} R_{2} x\left(t_{2}\right)\right| \leq & \frac{L_{2} \Gamma(n-q)}{\left|1-\lambda_{2} \xi^{n-q-1}\right| \Gamma\left(n-\beta_{h}\right) \Gamma\left(\beta_{0}-q+1\right)}\left(t_{2}^{n-\beta_{h}-1}-t_{1}^{n-\beta_{h}-1}\right) \\
& +\frac{L_{2}\left|\lambda_{2}\right| \Gamma(n-q) \eta^{\beta_{0}-q}}{\left|1-\lambda_{2} \xi^{n-q-1}\right| \Gamma\left(n-\beta_{h}\right) \Gamma\left(\beta_{0}-q+1\right)}\left(t_{1}^{n-\beta_{h}-1}-t_{2}^{n-\beta_{h}-1}\right) .
\end{aligned}
$$

As $t_{2} \rightarrow t_{1}$, the right-hand sides of the inequalities (3.33), (3.34), (3.35) and (3.36) tend to zero. Then, as a consequence of the steps $\left(a^{*}\right)$ and $\left(b^{*}\right)$ and by Arzela-Ascoli theorem, we conclude that $R$ is completely continuous.

$\left(3^{*}:\right)$ Finally, we prove that $T$ is a contraction mapping: Let $(x, y)\left(x_{1}, y_{1}\right) \in X \times Y$. Then, for each $t \in J$ and by $(H 2)$, we have

$$
\left|T_{1} y(t)-T_{1} y_{1}(t)\right| \leq \frac{\left(\omega_{0}+\omega_{1}+\cdots+\omega_{n-1}\right)\left[\left\|y-y_{1}\right\|+\left\|D^{\alpha_{1}} y-D^{\alpha_{1}} y_{1}\right\|+\cdots+\left\|D^{\alpha_{n-1}} y-D^{\alpha_{n-1}} y_{1}\right\|\right]}{\Gamma\left(\alpha_{0}+1\right)} .
$$

On the other hand, for all $k=1,2, \ldots, n-1$, we have

$\left|D^{\alpha_{k}} T_{1} y(t)-D^{\alpha_{k}} T_{1} y_{1}(t)\right| \leq \frac{\left(\omega_{0}+\omega_{1}+\cdots+\omega_{n-1}\right)\left[\left\|y-y_{1}\right\|+\left\|D^{\alpha_{1}} y-D^{\alpha_{1}} y_{1}\right\|+\cdots+\left\|D^{\alpha_{n-1}} y-D^{\alpha_{n-1}} y_{1}\right\|\right]}{\Gamma\left(\alpha_{0}-\alpha_{k}+1\right)}$.

By (3.37) and (3.38), we obtain

$$
\begin{aligned}
\left|T_{1} y(t)-T_{1} y_{1}(t)\right| \leq & {\left[\frac{\omega}{\Gamma\left(\alpha_{0}+1\right)}+\omega \sum_{k=1}^{n-1} \frac{1}{\Gamma\left(\alpha_{0}-\alpha_{k}+1\right)}\right] } \\
& \times\left(\left\|y-y_{1}\right\|+\left\|D^{\alpha_{1}} y-D^{\alpha_{1}} y_{1}\right\|+\cdots+\left\|D^{\alpha_{n-1}} y-D^{\alpha_{n-1}} y_{1}\right\|\right) .
\end{aligned}
$$


Analogously, for all $h=1,2, \ldots, n-1$, we can get

$$
\begin{aligned}
\left|T_{2} x(t)-T_{2} x_{1}(t)\right| \leq & {\left[\frac{\varpi}{\Gamma\left(\beta_{0}+1\right)}+\varpi \sum_{h=1}^{n-1} \frac{1}{\Gamma\left(\beta_{0}-\beta_{h}+1\right)}\right] } \\
& \times\left(\left\|x-x_{1}\right\|+\left\|D^{\beta_{1}} x-D^{\beta_{1}} x_{1}\right\|+\cdots+\left\|D^{\beta_{n-1}} x-D^{\beta_{n-1}} x_{1}\right\|\right) .
\end{aligned}
$$

Combining (3.39) and (3.40) yields

$$
\begin{aligned}
& \left\|T(x, y)(t)-T\left(x_{1}, y_{1}\right)(t)\right\|_{X \times Y} \leq\left(\left\|\left(x-x_{1}, y-y_{1}\right)\right\|_{X \times Y}\right) \\
& \quad \times\left[\left(\frac{1}{\Gamma\left(\alpha_{0}+1\right)}+\sum_{k=1}^{n-1} \frac{1}{\Gamma\left(\alpha_{0}-\alpha_{k}+1\right)}\right) \omega+\left(\frac{1}{\Gamma\left(\beta_{0}+1\right)}+\sum_{h=1}^{n-1} \frac{1}{\Gamma\left(\beta_{0}-\beta_{h}+1\right)}\right) \varpi\right]
\end{aligned}
$$

Using the condition (3.24), we deduce that $T$ is a contraction mapping.

As a consequence of Krasnoselskii's fixed point theorem we deduce that $\phi$ has a fixed point which is a solution of the problem (1.1) .

We give also the following two corollaries.

Corollary 3.1. Assume that $\eta^{n-p-1} \neq \frac{1}{\lambda_{1}}, \xi^{n-q-1} \neq \frac{1}{\lambda_{2}}$ and there exist non negative real numbers $\theta_{i}, \Lambda_{i}, i=0,1, \ldots, n-1$ such that for all $t \in[0,1]$ and $\left(x_{0}, x_{1}, \ldots, x_{n-1}\right)$, $\left(y_{0}, y_{1}, \ldots, y_{n-1}\right) \in \mathbb{R}^{n}$, we have $\left|f_{1}\left(t, x_{0}, x_{1}, \ldots, x_{n-1}\right)-f_{1}\left(t, y_{0}, y_{1}, \ldots, y_{n-1}\right)\right| \leq$ $\theta_{0}\left|x_{0}-y_{0}\right|+\cdots+\theta_{n-1}\left|x_{n-1}-y_{n-1}\right|$ and $\left|f_{2}\left(t, x_{0}, x_{1}, \ldots, x_{n-1}\right)-f_{2}\left(t, y_{0}, y_{1}, \ldots, y_{n-1}\right)\right|$ $\leq \Lambda_{0}\left|x_{0}-y_{0}\right|+\cdots+\Lambda_{n-1}\left|x_{n-1}-y_{n-1}\right|$. If

$$
\left(N_{0}+\sum_{k=1}^{n-1} N_{k}\right)\left(\theta_{0}+\cdots+\theta_{n-1}\right)+\left(M_{0}+\sum_{h=1}^{n-1} M_{h}\right)\left(\Lambda_{0}+\cdots+\Lambda_{n-1}\right)<1,
$$

then the fractional system (1.1) has a unique solution on $J$.

Corollary 3.2. Assume that $(H 1)$ holds and $\eta^{n-p-1} \neq \frac{1}{\lambda_{1}}, \xi^{n-q-1} \neq \frac{1}{\lambda_{2}}$. If there exist $k_{1}>0$ and $k_{2}>0$, such that $f_{1} \leq k_{1}, f_{2} \leq k_{2}$ on $J \times \mathbb{R}^{n}$ then, the coupled system (1.1) has at least a solution on $J$.

\section{EXAmples}

Example 4.1. Consider the following fractional differential system, where $t \in[0,1]$

$$
\left\{\begin{array}{l}
D^{\frac{7}{2}} x(t)=\frac{|y(t)|+\left|D^{\frac{9}{4}} y(t)\right|+\left|D^{\frac{3}{2}} y(t)\right|+\left|D^{\frac{1}{2}} y(t)\right|}{\left(t^{2}+32 \pi\right)\left(e^{t}+|y(t)|+\left|D^{\frac{9}{4}} y(t)\right|+\left|D^{\frac{3}{2}} y(t)\right|+\left|D^{\frac{1}{2}} y(t)\right|\right)}+\cosh \left(2+t^{2}\right), \\
D^{\frac{11}{3}} y(t)=\frac{\sin x(t)+\sin D^{\frac{5}{2}} x(t)+\sin D^{\frac{6}{5}} x(t)+\sin D^{\frac{4}{5}} x(t)}{16\left(\pi t^{2}+1\right)}+\arctan (1+t), \\
x(0)=\sqrt{2}, x^{\prime}(0)=x^{\prime \prime}(0)=0, D^{\frac{1}{2}} x(1)=\frac{4}{5} D^{\frac{1}{2}} x\left(\frac{3}{4}\right), \\
y(0)=\sqrt{3}, y^{\prime}(0)=y^{\prime \prime}(0)=0, D^{\frac{1}{3}} y(1)=\frac{6}{7} D^{\frac{1}{3}} y\left(\frac{2}{5}\right) .
\end{array}\right.
$$


We have

$$
\begin{aligned}
f_{1}\left(t, x_{1}, x_{2}, x_{3}, x_{4}\right)= & \frac{\left|x_{1}\right|+\left|x_{2}\right|+\left|x_{3}\right|+\left|x_{4}\right|}{\left(t^{2}+32 \pi\right)\left(e^{t}+\left|x_{1}\right|+\left|x_{2}\right|+\left|x_{3}\right|+\left|x_{4}\right|\right)} \\
& +\cosh \left(2+t^{2}\right) \\
f_{2}\left(t, x_{1}, x_{2}, x_{3}, x_{4}\right)= & \frac{\sin \left(x_{1}\right)+\sin \left(x_{2}\right)+\sin \left(x_{3}\right)+\sin \left(x_{4}\right)}{16\left(\pi t^{2}+1\right)} \\
& +\arctan (1+t)
\end{aligned}
$$

where $t \in[0,1], x_{1}, x_{2}, x_{3}, x_{4} \in \mathbb{R}$.

Let $t \in[0,1]$ and $\left(x_{0}, x_{1}, x_{2}, x_{3}\right),\left(y_{0}, y_{1}, y_{2}, y_{3}\right) \in \mathbb{R}^{4}$. Then

$$
\begin{aligned}
\left|f_{1}\left(t, x_{0}, x_{1}, x_{2}, x_{3}\right)-f_{1}\left(t, y_{0}, y_{1}, y_{2}, y_{3}\right)\right| & \leq \frac{1}{\left(t^{2}+32 \pi\right)}\left(\left|x_{0}-y_{0}\right|+\left|x_{1}-y_{1}\right|\right. \\
& \left.+\left|x_{2}-y_{2}\right|+\left|x_{3}-y_{3}\right|\right), \\
\left|f_{2}\left(t, x_{0}, x_{1}, x_{2}, x_{3}\right)-f_{2}\left(t, y_{0}, y_{1}, y_{2}, y_{3}\right)\right| \leq & \frac{1}{16\left(\pi t^{2}+1\right)}\left(\left|x_{0}-y_{0}\right|+\left|x_{1}-y_{1}\right|\right. \\
& \left.+\left|x_{2}-y_{2}\right|+\left|x_{3}-y_{3}\right|\right) .
\end{aligned}
$$

We can take $a_{i}(t)=\frac{1}{\left(t^{2}+32 \pi\right)}, b_{i}(t)=\frac{1}{16\left(\pi t^{2}+1\right)}, i=0,1,2,3$. Then $\omega_{i}=\sup _{t \in[0,1]} a_{i}(t)$ $=\frac{1}{32 \pi}, \varpi_{i}=\sup _{t \in[0,1]} b_{i}(t)=\frac{1}{16}, i=0,1,2,3$ and $N_{0}=0.288269, N_{1}=2.203434$, $N_{2}=1.413119, N_{3}=0.615229, \omega=\frac{1}{8 \pi}, M_{0}=0.085713, M_{1}=1.124108, M_{2}=$ $0.322689, M_{3}=0.203869, \varpi=\frac{1}{4}$. We also have

$$
\left(N_{0}+\sum_{k=1}^{n-1} N_{k}\right) \omega+\left(M_{0}+\sum_{h=1}^{n-1} M_{h}\right) \varpi=0.179938+0.434094=0.614032<1 .
$$

Hence, by Theorem 3.1, the system (4.1) has a unique solution on [0,1] .

Example 4.2. The second example is the following system:

$$
\left\{\begin{array}{l}
D^{\frac{10}{3}} x(t)=\frac{\cos y(t)+\sin \left(D^{\frac{10}{4}} y(t)+D^{\frac{9}{7}} y(t)\right)}{\pi e^{t}+15}, t \in[0,1], \\
D^{\frac{14}{4}} y(t)=\frac{\sin x(t)+\cos \left(D^{\frac{5}{2}} x(t)+D^{\frac{4}{3}} x(t)\right)}{t^{2}+20 \pi}, t \in[0,1] \\
x(0)=3, x^{\prime}(0)=x^{\prime \prime}(0)=0, D^{\frac{4}{5}} x(1)=\frac{3}{4} D^{\frac{4}{5}} x\left(\frac{1}{3}\right), \\
y(0)=\sqrt{5}, y^{\prime}(0)=y^{\prime \prime}(0)=0, D^{\frac{9}{8}} y(1)=\frac{2}{3} D^{\frac{9}{8}} y\left(\frac{2}{5}\right) .
\end{array}\right.
$$

We have

$$
\begin{aligned}
& f_{1}(t, x, y, z)=\frac{\cos x+\sin (y+z)}{\pi e^{t}+15}, t \in[0,1],(x, y, z) \in \mathbb{R}^{3}, \\
& f_{2}(t, x, y, z)=\frac{\sin x+\cos (y+z)}{20 \pi+t^{2}}, t \in[0,1],(x, y, z) \in \mathbb{R}^{3} .
\end{aligned}
$$

Let $x, y, z \in \mathbb{R}$ and $t \in[0,1]$. Then $\left|f_{1}(t, x, y, z)\right| \leq \frac{2}{\pi e^{t}+15},\left|f_{2}(t, x, y, z)\right| \leq \frac{2}{t^{2}+20 \pi}$. So, we take $l_{1}(t)=\frac{2}{\pi e^{t}+15}, l_{2}(t)=\frac{2}{t^{2}+20 \pi}$. Then, $L_{1}=\frac{2}{\pi+15}, L_{2}=\frac{1}{10 \pi}$. By Theorem 3.2, the system (4.2) has at least one solution on $[0,1]$. 
Example 4.3. Our third example is the following:

$$
\left\{\begin{array}{l}
D^{\frac{17}{5}} x(t)=\frac{e^{-t^{2}}\left(|y(t)|+\left|D^{\frac{15}{6}} y(t)\right|+\left|D^{\frac{11}{7}} y(t)\right|+\left|D^{\frac{2}{3}} y(t)\right|\right)}{\left(e^{t^{2}}+20 \pi\right)\left(2 \pi e^{t^{2}}+|y(t)|+\left|D^{\frac{15}{6}} y(t)\right|+\left|D^{\frac{11}{7}} y(t)\right|+\left|D^{\frac{2}{3}} y(t)\right|\right)}+\cos \left(2+t^{2}\right), \\
D^{\frac{15}{4}} y(t)=\frac{|x(t)|+\left|D^{\frac{11}{5}} x(t)\right|+\left|D^{\frac{4}{3}} x(t)\right|+\left|D^{\frac{1}{2}} x(t)\right|}{\left(\pi e^{t}+18\right)\left(e^{-t}+|x(t)|+\left|D^{\frac{11}{5}} x(t)\right|+\left|D^{\frac{4}{3}} x(t)\right|+\left|D^{\frac{1}{2}} x(t)\right|\right)}+\ln \left(2+t^{2}\right), \\
x(0)=2, x^{\prime}(0)=x^{\prime \prime}(0)=0, D^{\frac{5}{4}} x(1)=\frac{5}{6} D^{\frac{5}{4}} x\left(\frac{2}{5}\right), \\
y(0)=3, y^{\prime}(0)=y^{\prime \prime}(0)=0, D^{\frac{3}{2}} y(1)=\frac{7}{8} D^{\frac{3}{2}} y\left(\frac{3}{5}\right),
\end{array}\right.
$$

where $t \in[0,1]$. For this example, for $t \in[0,1], x_{1}, x_{2}, x_{3}, x_{4} \in \mathbb{R}$ we have

$$
\begin{aligned}
f_{1}\left(t, x_{1}, x_{2}, x_{3}, x_{4}\right)= & \frac{e^{-t^{2}}\left(\left|x_{1}\right|+\left|x_{2}\right|+\left|x_{3}\right|+\left|x_{4}\right|\right)}{\left(e^{t^{2}}+20 \pi\right)\left(2 \pi e^{t^{2}}+\left|x_{1}\right|+\left|x_{2}\right|+\left|x_{3}\right|+\left|x_{4}\right|\right)} \\
& +\cos \left(2+t^{2}\right) \\
f_{2}\left(t, x_{1}, x_{2}, x_{3}, x_{4}\right)= & \frac{\left|x_{1}\right|+\left|x_{2}\right|+\left|x_{3}\right|+\left|x_{4}\right|}{\left(\pi e^{t}+18\right)\left(e^{-t}+\left|x_{1}\right|+\left|x_{2}\right|+\left|x_{3}\right|+\left|x_{4}\right|\right)} \\
& +\ln \left(2+t^{2}\right)
\end{aligned}
$$

Taking $x_{0}, x_{1}, x_{2}, x_{3}, y_{0}, y_{1}, y_{2}, y_{3} \in \mathbb{R}, t \in[0,1]$, we have

$$
\begin{aligned}
\left|f_{1}\left(t, x_{0}, x_{1}, x_{2}, x_{3}\right)-f_{1}\left(t, y_{0}, y_{1}, y_{2}, y_{3}\right)\right| \leq & \frac{e^{-t^{2}}}{e^{t^{2}}+20 \pi}\left(\left|x_{0}-y_{0}\right|+\left|x_{1}-y_{1}\right|\right. \\
& \left.+\left|x_{2}-y_{2}\right|+\left|x_{3}-y_{3}\right|\right) \\
\left|f_{2}\left(t, x_{0}, x_{1}, x_{2}, x_{3}\right)-f_{2}\left(t, y_{0}, y_{1}, y_{2}, y_{3}\right)\right| \leq & \frac{1}{\pi e^{t}+18}\left(\left|x_{0}-y_{0}\right|+\left|x_{1}-y_{1}\right|\right. \\
& \left.+\left|x_{2}-y_{2}\right|+\left|x_{3}-y_{3}\right|\right) .
\end{aligned}
$$

Hence, $a_{i}(t)=\frac{e^{-t^{2}}}{\left(e^{t^{2}+20 \pi}\right)}, b_{i}(t)=\frac{1}{\left(\pi e^{t}+18\right)}, i=0, \ldots, 3$.

Then, $\omega_{i}=\sup _{t \in[0,1]} a_{i}(t)=\frac{1}{1+20 \pi}, \varpi_{i}=\sup _{t \in[0,1]} b_{i}(t)=\frac{1}{\pi+18}, i=0, . ., 3$ and for $k, h \in\{=1,2,3\}$, we have

$$
\begin{aligned}
& \frac{1}{\Gamma\left(\alpha_{0}+1\right)}+\sum_{k=1}^{n-1} \frac{1}{\Gamma\left(\alpha_{0}-\alpha_{k}+1\right)}=1.951303 \\
& \frac{1}{\Gamma\left(\beta_{0}+1\right)}+\sum_{h=1}^{n-1} \frac{1}{\Gamma\left(\beta_{0}-\beta_{h}+1\right)}=1.236325 .
\end{aligned}
$$

We also have

$$
\left(\frac{1}{\Gamma\left(\alpha_{0}+1\right)}+\sum_{k=1}^{n-1} \frac{1}{\Gamma\left(\alpha_{0}-\alpha_{k}+1\right)}\right) \omega+\left(\frac{1}{\Gamma\left(\beta_{0}+1\right)}+\sum_{h=1}^{n-1} \frac{1}{\Gamma\left(\beta_{0}-\beta_{h}+1\right)}\right) \varpi=0.3562179<1 .
$$

By Theorem 3.3, we can state that the problem (4.3) has at least one solution on $[0,1]$. 


\section{REFERENCES}

[1] A. Anber, S. Belarbi, Z. Dahmani, New existence and uniqueness results for fractional differential equations, An. St. Univ. Ovidius Constanta. Vol. 21(3), (2013) 33-41.

[2] M. E. Bengrine, Z. Dahmani, Boundary value problems for fractional differential equations, Int. J. Open problems compt. Math. 5(4), (2012) 7-15.

[3] Z. Cui, P. Yu, Z. Mao, Existence of solutions for nonlocal boundary value problems of nonlinear fractional differential equations. Advances in Dynamical Systems and Applications 7(1), (2012) $31-40$.

[4] K. Diethelm, N. J. Ford, Analysis of fractiona differential equations, J. Math. Anal. Appl. 265(2), (2002) 229-248.

[5] A. M. A. El-Sayed, Nonlinear functional differential equations of arbitrary orders, Nonlinear Anal. 33(2), (1998) 181-186.

[6] W. Feng, S. Sun, Z. Han, Y. Zhao, Existence of solutions for a singular system of nonlinear fractional differential equations, Comput. Math. Appl. 62 (2011) 1370-1378.

[7] M. Gaber, M. G. Brikaa, Existence results for a coupled system of nonlinear fractional differential equation with three-point boundary conditions. Journal of Fractional Calculus and Applications. 3(21), (2012.) 1-10.

[8] Z. Guo, M. Liu, On solutions of a system of higher-order nonlinear fractional differential equations. Bulletin of Mathematical Analysis and Applications 3(4), (2011) 59-68.

[9] M. Houas, Z. Dahmani, New fractional results for a boundary value problem with caputo derivative, Int. J. Open problems compt. Math. 6(2), (2013) 30-42.

[10] M. Houas, Z. Dahmani, M. Benbachir, New results for a boundary value problem for differential equations of arbitrary order, International Journal of Modern Mathematical Sciences 7(2), (2013) 195-211.

[11] M. Houas, Z. Dahmani, New results for a Caputo boundary value problem, American Journal of Computational and Applied Mathematics 3(3), (2013) 143-161.

[12] M. Houas, Z. Dahmani, New results for a coupled system of fractional differential equations, Facta Universitatis, Series Mathematics and Informatics, Accepted paper, 2013.

[13] Z. Hu, W. Liu, T. Chen, Existence solutions for a coupled system of fractional differential equations at resonance, Boundary Value Problems 98 (2012) 1-13.

[14] A. A. Kilbas, S. A. Marzan, Nonlinear differential equation with the Caputo fraction derivative in the space of continuously differentiable functions, Differ. Equ. 41(1), (2005) 84-89.

[15] V. Lakshmikantham, A. S. Vatsala, Basic theory of fractional differential equations, Nonlinear Anal. 69(8), (2008) 2677-2682.

[16] J. Liang, Z. Liu, X. Wang, Solvability for a couple system of nonlinear fractional differential equations in a banach space, Fractional Calculus and Applied Analysis 16(1), (2013) 51-63.

[17] R. Liu, C. Kou, X. Xie, Existence Results for a Coupled System of Nonlinear Fractional Boundary Value Problems at Resonance, Mathematical Problems in Engineering, Vol. 2013, Art. ID 267386, (2013) 1-9.

[18] F. Mainardi, Fractional Calculus-Some basic problem in continuum and statistical mechanics. Fractals and fractional calculus in continuum mechanics, Springer, Vienna, 1997 291-348.

[19] J. Nieto, J. Pimente, Positive solutions of a fractional thermostat model, Boundary Value Problems Art. 5 (2013) 1-11.

[20] I. Podlubny, I. Petras, B. M. Vinagre, P. O'leary, L. Dorcak, Analogue realizations of fractionalorder controllers. Fractional order calculus and its applications, Nonlinear Dynam. 29(4), (2002) 281-296.

[21] S. Ntouyas, M. Obaid, A coupled system of fractional differential equation with nonlocal integral boundary conditions, Boundary Value Problems, 130 (2012) 1-8. 
[22] G. Wang, R. P. Agarwal, A. Cabada, Existence results and monotone iterative technique for systems of nonlinear fractional differential equations, Applied Mathematics Letters 25 (2012) 1019-1024.

[23] W. Yang, Positive solutions for a coupled system of nonlinear fractional differential equations with integral boundary conditions, Comput. Math. Appl. 63, (2012) 288-297.

[24] W. Yang, Three-point boundary value problems for a coupled system of nonlinear fractional differential equations, J. Appl. Math. Informatics 30(5-6), (2012) 773-785.

[25] Y. Zhang, Z. Bai, T. Feng: Existence results for a coupled system of nonlinear fractional threepoint boundary value problems at resonance, Comput. Math. Appl. 61 (2011) 1032-1047.

[26] Y. Liu, B. Ahmad, R. P. Agarwal, Existence of solutions for a coupled system of nonlinear fractional differential equations with fractional boundary conditions on the half-line, Advances in Difference Equations, Vol. 46 (2013) 1-19.

[27] X. Zhang, C. Zhu, Z. Wu, Solvability for a coupled system of fractional differential equations with impulses at resonance, Boundary Value Problems, Vol. 80 (2013) 1-23.

${ }^{1}$ Department of Mathematics, University of Khemis MiLiana, ALGERIA

E-mail address: houasmed@yahoo.fr

${ }^{2}$ LPAM,

FACULTY SEI,

UMAB Mostaganem,

AlgERIA

E-mail address: zzdahmani@yahoo.fr 